\title{
On some densities in the set of permutations
}

\author{
Eugenijus Manstavičius * \\ Department of Mathematics and Informatics, Vilnius University \\ and \\ Institute of Mathematics and Informatics \\ Vilnius, Lithuania \\ eugenijus.manstavicius@mif.vu.It
}

Submitted: Aug 24, 2008; Accepted: Jun 26, 2010; Published: Jul 10, 2010

Mathematics Subject Classifications: 60C05, 05A16

\begin{abstract}
The asymptotic density of random permutations with given properties of the $k$ th shortest cycle length is examined. The approach is based upon the saddle point method applied for appropriate sums of independent random variables.
\end{abstract}

\section{Introduction}

Let $n \in \mathbf{N}, \mathbf{S}_{n}$ be the symmetric group of permutations acting on the set $\{1,2, \ldots, n\}$, and $\mathbf{S}:=\mathbf{S}_{1} \cup \mathbf{S}_{2} \cdots$ Set $\nu_{n}$ for the uniform probability measure on $\mathbf{S}_{n}$. By $\nu_{n}(A):=\nu_{n}\left(A \cap \mathbf{S}_{n}\right)$, we trivially extend it for all subsets of $\mathbf{S}$. If the limit

$$
\lim _{n \rightarrow \infty} \nu_{n}(A)=: d(A), \quad A \subset \mathbf{S}
$$

exists, $d(A)$ can be called the asymptotic density of $A$. Let $\mathcal{S} \subset \mathbf{S}$ be the class of $A$ having an asymptotic density $d(A)$. The triple $\{\mathbf{S}, \mathcal{S}, d\}$ is far from being a probability space. However, the behavior of $d\left(A_{m}\right)$ for some specialized subsets $A_{m} \in \mathcal{S}$ as $m \rightarrow \infty$ are worth to be investigated. In this paper, we demonstrate that by taking sets connected to the ordered statistics of different cycle lengths.

Recall that each $\sigma \in \mathbf{S}_{n}$ can be uniquely (up to the order) written as a product of independent cycles. Let $k_{j}(\sigma) \geqslant 0$ be the number of cycles of length $j, 1 \leqslant j \leqslant n$, in such decomposition. The structure vector is defined as

$$
\bar{k}(\sigma):=\left(k_{1}(\sigma), \ldots, k_{n}(\sigma)\right) .
$$

${ }^{*}$ The final version of this paper was written during the author's stay at Institute of Statistical Science of Academia Sinica, Taipei. We gratefully acknowledge the support and thank Professors Hsien-Kuei Hwang and Vytas Zacharovas for the warm hospitality. 
Set $\ell(\bar{k}):=1 k_{1}+\cdots+n k_{n}$, where $\bar{k}:=\left(k_{1}, \ldots, k_{n}\right) \in \mathbf{Z}_{+}^{n}$, then $\ell(\bar{k}(\sigma))=n$. Moreover, if $\ell(\bar{k})=n$, then the set $\left\{\sigma \in \mathbf{S}_{n}: \bar{k}(\sigma)=\bar{k}\right\}$ agrees with the class of conjugate permutations in $\mathbf{S}_{n}$. If $\xi_{j}, j \geqslant 1$, are independent Poisson random variables (r.vs) given on some probability space $\{\Omega, \mathcal{F}, P\}, \mathbf{E} \xi_{j}=1 / j$, and $\bar{\xi}:=\left(\xi_{1}, \ldots, \xi_{n}\right)$, then $[2]$

$$
\nu_{n}(\bar{k}(\sigma)=\bar{k})=\mathbf{1}\{\ell(\bar{k})=n\} \prod_{j=1}^{n} \frac{1}{j^{k_{j}} k_{j} !}=P(\bar{\xi}=\bar{k} \mid \ell(\bar{\xi})=n),
$$

where 1 denotes the indicator function. Moreover,

$$
\left(k_{1}(\sigma), \ldots k_{n}(\sigma), 0, \ldots,\right) \stackrel{\nu_{n}}{\Rightarrow}\left(\xi_{1}, \ldots, \xi_{n}, \xi_{n+1}, \ldots\right)
$$

in the sense of convergence of the finite dimensional distributions. Here and in what follows we assume that $n \rightarrow \infty$. The so-called Fundamental Lemma sheds more light than (1). We state it as the following estimate of the total variation distance. If $\bar{k}(\sigma)_{r}:=$ $\left(k_{1}(\sigma), \ldots, k_{r}(\sigma)\right)$ and $\bar{\xi}_{r}:=\left(\xi_{1}, \ldots, \xi_{r}\right)$, then $[1]$

$$
\frac{1}{2} \sum_{\bar{k} \in \mathbf{Z}_{+}^{r}}\left|\nu_{n}\left(\bar{k}(\sigma)_{r}=\bar{k}\right)-P\left(\bar{\xi}_{r}=\bar{k}\right)\right|=R(n / m)
$$

for $1 \leqslant r \leqslant n$. Here and in the sequel $R(u)$ denotes an error term which has the upper bound

$$
R(u)=\mathrm{O}\left(\mathrm{e}^{-u \log u+\mathrm{O}(u)}\right)
$$

with some absolute constants in $\mathrm{O}(\cdot)$.

In the recent decade, a lot of investigations were devoted to the limit distributions of values of additive functions with respect to $\nu_{n}$. Given a real two dimensional sequence $\left\{h_{j}(k)\right\}, j, k \geqslant 1, h_{j}(0) \equiv 0$, such a function is defined as

$$
h(\sigma)=\sum_{j \leqslant n} h_{j}\left(k_{j}(\sigma)\right) .
$$

The relevant references can be found in [2], [12], [15] and other papers. The family of additive functions

$$
s(\sigma, y)=\sum_{j \leqslant y} \mathbf{1}\left\{k_{j}(\sigma) \geqslant 1\right\}, \quad 1 \leqslant y \leqslant n,
$$

is closely related to the ordered statistics

$$
j_{1}(\sigma)<j_{2}(\sigma)<\cdots<j_{s}(\sigma)
$$

of different cycle lengths appearing in the decomposition (1). Now $s:=s(\sigma, n)$ counts all such lengths. We have $s\left(\sigma, j_{k}(\sigma)\right)=k$ for each $1 \leqslant k \leqslant s$. The last relation and the laws of iterated logarithm for $s(\sigma, m)$ led [14] to the following result.

Denote $L u=\log \max \{u, e\}=L_{1} u, \ldots, L_{r} u=L\left(L_{r-1} u\right)$ for $u \in \mathbf{R}$. For $0<\delta<1$ and $r \geqslant 2$, set

$$
\beta_{r k}(1 \pm \delta)=\left(2 k\left(L_{2} k+\frac{3}{2} L_{3} k+L_{4} k+\cdots+(1 \pm \delta) L_{r} k\right)\right)^{1 / 2}
$$


Theorem ([14]). For arbitrary $0<\delta<1$ and $r \geqslant 2$, we have

$$
\lim _{n_{1} \rightarrow \infty} \varlimsup_{n \rightarrow \infty} \nu_{n}\left(\max _{n_{1} \leqslant k \leqslant s} \frac{\left|\log j_{k}(\sigma)-k\right|}{\beta_{r k}(1+\delta)} \geqslant 1\right)=0
$$

and

$$
\lim _{n_{1} \rightarrow \infty} \underline{\lim }_{n \rightarrow \infty} \nu_{n}\left(\max _{n_{1} \leqslant k \leqslant s} \frac{\left|\log j_{k}(\sigma)-k\right|}{\beta_{r k}(1-\delta)} \geqslant 1\right)=1 .
$$

Thus, we may say that "for almost all $\sigma \in \mathbf{S}_{n}$ "

$$
\left|\log j_{k}(\sigma)-k\right| \leqslant \beta_{r k}(1+\delta)
$$

uniformly in $k, n_{1} \leqslant k \leqslant s$, where $n_{1} \rightarrow \infty$ arbitrarily slowly. This assertion is sharp in the sense that we can not change $\delta$ by $-\delta$. It can be compared with the following corollary of the invariance principle (see [3], [14]) where the convergence of distributions is examined.

Theorem ([14]). Uniformly in $x \in \mathbf{R}$,

$$
\nu_{n}\left(\max _{k \leqslant s}\left|\log j_{k}(\sigma)-k\right| \leqslant x \sqrt{\log n}\right)=\frac{1}{\sqrt{2 \pi}} \sum_{l \in \mathbf{Z}}(-1)^{l} \int_{-x}^{x} \mathrm{e}^{-(u-2 l x)^{2} / 2} \mathrm{~d} u+\mathrm{o}(1) .
$$

Instead of $j_{k}(\sigma), 1 \leqslant k \leqslant s$, one can deal with the sequence

$$
J_{1}(\sigma) \leqslant J_{2}(\sigma) \leqslant \cdots \leqslant J_{w}(\sigma)
$$

of all cycle lengths appearing in the decomposition of $\sigma$. The behavior of these ordered statistics is similar, however, some technical differences do arise in their analysis. Section 3.2 of the paper by D. Panario and B. Richmond [16] contains rather complicated asymptotical formulas for $\nu_{n}\left(J_{k}(\sigma)=m\right)$ as $m, n \rightarrow \infty$ if $k$ is fixed. We are more interested into the case when $k$ is unbounded therefore we now include V.F. Kolchin's result for the so-called middle region.

Theorem ([9]). Let $0<\alpha<1$ be fixed, $k=\alpha \log n+\mathrm{o}(\sqrt{\log n})$, and $n \rightarrow \infty$. Then

$$
\nu_{n}\left(\log J_{k}(\sigma) \leqslant k+x \sqrt{k}\right)=\frac{1}{\sqrt{2 \pi}} \int_{-\infty}^{x} \mathrm{e}^{-u^{2} / 2} \mathrm{~d} u+\mathrm{o}(1)=: \Phi(x)+\mathrm{o}(1) .
$$

Despite such variety of results, the frequency

$$
\nu_{n}\left(j_{k}(\sigma)=m\right)=\nu_{n}\left(k_{m}(\sigma) \geqslant 1, s(\sigma, m-1)=k-1\right),
$$

where $1 \leqslant k \leqslant m \leqslant n$, can further be examined. Observe that the event under frequency is described in terms of the first $m$ components $k_{j}(\sigma)$ of the structure vector. If $m=$ $\mathrm{o}(n)$, then by (2) its frequency can be approximated by an appropriate probability for independent random variables $\xi_{j}, 1 \leqslant j \leqslant m$.

Introduce the independent Bernoulli r. vs $\eta_{j}, j \geqslant 1$, such that

$$
P\left(\eta_{j}=1\right)=1-\mathrm{e}^{-1 / j}=1-P\left(\eta_{j}=0\right)
$$

and set $X_{y}=\sum_{j \leqslant y} \eta_{j}$ where $1 \leqslant y \leqslant n$. 
Proposition 1. For $1 \leqslant k \leqslant m=\mathrm{o}(n)$,

$$
\begin{aligned}
& \nu_{n}\left(j_{k}(\sigma)=m\right)=P\left(\eta_{m}=1\right) P\left(X_{m-1}=k-1\right)+R(n / m) \\
& =\left(1-\mathrm{e}^{-1 / m}\right) \exp \left\{-\sum_{j<m} \frac{1}{j}\right\} \sum_{1 \leqslant j_{1}<\cdots<j_{k-1}<m} \prod_{r=1}^{k-1}\left(\mathrm{e}^{1 / j_{r}}-1\right) \\
& \quad+R(n / m),
\end{aligned}
$$

where the remainder term is estimated in (3).

This simple corollary following from (2) motivates our interest to the probabilities $P_{m}(k):=P\left(X_{m}=k\right)$ if $k \geqslant 0$ and $m \geqslant 3$. Of course, we can exclude the trivial cases $P_{m}(k)=0$ if $k>m$,

$$
P_{m}(0)=\exp \left\{-\sum_{j \leqslant m} \frac{1}{j}\right\} \sim \frac{\mathrm{e}^{-\gamma}}{m}
$$

and

$$
P_{m}(m)=\prod_{j \leqslant m}\left(1-\mathrm{e}^{-1 / j}\right) \sim C_{0} \frac{\sqrt{P_{m}(0)}}{m !} .
$$

Here $m \rightarrow \infty, \gamma$ is the Euler constant, and

$$
C_{0}=\prod_{j \geqslant 1}\left(1+\frac{1}{2 ! j}+\frac{1}{3 ! j^{2}}+\cdots\right) \mathrm{e}^{-1 /(2 j)} .
$$

Contemporary probability theory provides a lot of local theorems for sums of independent Bernoulli r. vs. Since

$$
\begin{aligned}
\lambda_{m}:=\mathbf{E} X_{m} & =\sum_{j \leqslant m}\left(1-\mathrm{e}^{-1 / j}\right)=\log m+C+\mathrm{O}\left(\frac{1}{m}\right), \\
C & :=\gamma+\sum_{j \geqslant 1}\left(1-\mathrm{e}^{-1 / j}-\frac{1}{j}\right),
\end{aligned}
$$

and, similarly,

$$
\mathbf{V} a r X_{m}=\log m+C_{1}+\mathrm{O}\left(\frac{1}{m}\right),
$$

where $C_{1}$ is a constant, and $m \geqslant 3$, the results on the so-called large deviations imply the approximations for $P_{m}(k)$ in the region $k-\log m=\mathrm{o}(\log m)$ as $m \rightarrow \infty$ (see [17], Chapter VIII or [7]). H.-K. Hwang's work [8] as well as many others can be used in this zone. However, we still have a great terra incognita if $(1+\varepsilon) \log m \leqslant k \leqslant m-1$ where $\varepsilon>0$. The present paper sheds some light to it. First, we prove some new asymptotic formulas for $P_{m}(k)$ which are nontrivial outside the region of classical large deviations. Further, we apply them by inserting into the equalities given in Proposition 1 . The very idea goes back to the number-theoretical paper by P. Erdős and G. Tenenbaum [6]. 
We now introduce some notation. Denote

$$
F(z, m)=\sum_{0 \leqslant k \leqslant m} q_{k}(m) z^{k}:=\prod_{j \leqslant m}\left(1+\left(\mathrm{e}^{1 / j}-1\right) z\right), \quad z \in \mathbf{C} .
$$

Then $P_{m}(k)=q_{k}(m) / F(1, m)$. Let $\rho(t, m)$ satisfy the saddle point equation

$$
x \frac{F^{\prime}(x, m)}{F(x, m)}=\sum_{j \leqslant m} \frac{x}{a_{j}+x}=t, \quad a_{j}:=\left(\mathrm{e}^{1 / j}-1\right)^{-1}
$$

for $0 \leqslant t \leqslant m-1$. Set

$$
W(t)=\Gamma(t+1) t^{-t} \mathrm{e}^{t}
$$

if $t>0$ and $W(0)=1$, where $\Gamma(t)$ denotes the Euler gamma-function.

Theorem 1. Let $0<\varepsilon<1$ be arbitrary, $m \geqslant 3$, and $0^{0}:=1$. Then

$$
P_{m}(k)=\frac{F(\rho(k, m), m)}{F(1, m)} \frac{1}{\rho(k, m)^{k} W(k)}\left(1+\mathrm{O}\left(\frac{1}{\log m}\right)\right)
$$

uniformly in $0 \leqslant k \leqslant m^{1-\varepsilon}$.

Further analysis of the involved quantities leads to interesting simpler formulae. Set

$$
L(t, m)=\log \frac{m}{1+t / \log m} .
$$

Corollary. If $0 \leqslant k \leqslant m^{1-\varepsilon}$, then

$$
P_{m}(k)=\frac{1}{F(1, m)} \frac{L(k, m)^{k}}{k !} \exp \left\{\mathrm{O}\left(\frac{k}{\log m}\right)\right\},
$$

and

$$
P_{m}(k)=\frac{F(k / L(k, m), m)}{F(1, m)}\left(\frac{L(k, m)}{\mathrm{e}}\right)^{k} \frac{1}{k !} \exp \left\{\mathrm{O}\left(\frac{k}{\log ^{2} m}+\frac{1}{\log m}\right)\right\} .
$$

The first formula in the corollary implies an asymptotic expression only in the region $k=\mathrm{o}(\log m)$, however, it yields an effective estimate of $P_{m}(k)$ for $0 \leqslant k \leqslant m^{1-\varepsilon}$. The classical results for $k-\log m=\mathrm{o}(\log m)$ are hidden in the second one. Instead of going into the details of that, we return to the cycle lengths and exploit Proposition 1. Set

$$
d_{k}(m)=d\left(j_{k}(\sigma)=m\right)=\left(1-\mathrm{e}^{-1 / m}\right) P_{m-1}(k-1) .
$$

Theorem 2. Let $m \geqslant 3,0<\varepsilon<1$, and $1 \leqslant k \leqslant m^{1-\varepsilon}$. Then

$$
\frac{d_{k+1}(m)}{d_{k}(m)}=\frac{L(k, m)}{k}\left(1+\mathrm{O}\left(\frac{1}{\log m}\right)\right) \text {. }
$$

Moreover,

$$
\max _{1 \leqslant k \leqslant m} d_{k}(m)=\frac{1}{m \sqrt{2 \pi \log m}}\left(1+\mathrm{O}\left(\frac{1}{\log m}\right)\right)
$$

and the maximum is achieved at

$$
k=k_{m}=\log m+\mathrm{O}(1) .
$$


Having this and some other arguments in mind, we conjecture that $d_{k}(m)$ is unimodal for $1 \leqslant k \leqslant m$ and all $m \geqslant 3$.

Going further, we can exploit an idea from the renewal theory. The event $\left\{\sigma: j_{k}(\sigma)>\right.$ $y\}$ occurs if and only if $\sigma \in \mathbf{S}_{n}$ has less than $k$ cycles with lengths in [1,y]. Hence, by (2),

$$
d\left(j_{k}(\sigma)>y\right)=d(s(\sigma, y)<k)=P\left(X_{y}<k\right) .
$$

The last probability is traditionally examined as $y \rightarrow \infty$ and $k=k(y)$ belonging to specified regions. This can be exploited. For instance, applying formula (16) from [4], we have

$$
\sup _{k \geqslant 1}\left|d\left(j_{k}(\sigma)>y\right)-\Pi_{\lambda_{y}}(k)\right|=\frac{1}{2 \lambda_{y} \sqrt{2 \pi \mathrm{e}}} \sum_{j \leqslant y}\left(1-\mathrm{e}^{-1 / j}\right)^{2}+\mathrm{O}\left((\log y)^{-3 / 2}\right),
$$

where $\Pi_{\lambda_{y}}(\cdot)$ is the Poisson distribution with the parameter $\lambda_{y}$ defined above. The paper [4] and many other works published in the last decade provide even more exact approximations applicable for $d\left(j_{k}(\sigma)>y\right)$.

We now seek an asymptotical formula for it as $k \rightarrow \infty$, where $y=y(k)$ is a suitably chosen function of $k$. That may be ascribed to the renewal theory when the summands $\eta_{j}, j \geqslant 1$ in $X_{y}$ are independent but non-identically distributed. The next our result resembles in its form the Kolchin's theorem. The very idea goes back to the numbertheoretical paper [5] by J.-M. De Koninck and G. Tenenbaum.

Theorem 3. We have

$$
d\left(\log j_{k}(\sigma) \leqslant k+x \sqrt{k}\right)=\Phi(x)-\frac{x^{2}-1-3 C}{3 \sqrt{2 \pi k}} \mathrm{e}^{-x^{2} / 2}+\mathrm{O}\left(\frac{1}{k}\right)
$$

uniformly in $k \geqslant 1$ and $x \in \mathbf{R}$.

Finally, we observe that these results can be used to obtain asymptotical formulas for $\max _{m \geqslant k} d_{k}(m)$ as $k \rightarrow \infty$. We intend to discuss that in a forthcoming paper.

\section{The saddle point method}

Since $P_{m}(k)=q_{k}(m) / F(1, m)$, it suffices to analyze the Cauchy integral

$$
q_{k}(m)=\frac{1}{2 \pi i} \int_{|z|=\rho} \frac{F(z, m)}{z^{k+1}} \mathrm{~d} z .
$$

Similar but more complicated integrals have been the main task in the work on the number of permutations missing long cycles (see [10] and [11]). We now exploit this experience and the ideas coming from paper [6].

Henceforth let $k \geqslant 0, m \geqslant 3$, and $0<\varepsilon<1$. For $0 \leqslant t \leqslant m^{1-\varepsilon}$, we have $L:=$ $L(t, m) \asymp \log m$. Here and in the sequel the symbol $a \asymp b$ means $a \ll b$ and $b \ll a$ while 
$\ll$ is an analog of $\mathrm{O}(\cdot)$. The implicit constants in estimates depend at most on $\varepsilon$ therefore the remainder $\mathrm{O}(1 / L)$ is just a shorter form of $\mathrm{O}(1 / \log m)$.

Observe that the functions $x /\left(a_{j}+x\right), 1 \leqslant j \leqslant m-2$, are strictly increasing for $x \in[0, \infty)=\mathbf{R}_{+}$therefore the sum over $j$ varies from 0 to the value $m-0$. This proves the existence of a unique $\rho(t, m)>0$ for $0<t \leqslant m-1$ and $m \geqslant 3$. Moreover, $\rho(0, m)=0$. The main task of this section is to prove the following proposition.

Proposition 2. Let $\rho(k, m)$ be the solution to equation (5) for $t=k$. Then

$$
q_{k}(m)=\frac{F(\rho(k, m), m)}{\rho(k, m)^{k} W(k)}\left(1+\mathrm{O}\left(\frac{1}{L}\right)\right)
$$

uniformly in $0 \leqslant k \leqslant m^{1-\varepsilon}$. The function $W(t)$ has been defined in (6).

Firstly, we prove a few lemmas.

Lemma 1. For all $0 \leqslant t \leqslant m^{1-\varepsilon}$,

$$
\rho(t, m)=\frac{t}{L(t, m)}\left(1+\mathrm{O}\left(\frac{1}{L}\right)\right) .
$$

Proof. By the definition, using the inequalities $0<j-a_{j} \leqslant 2$ for all $j \geqslant 1$ and the abbreviation $\rho:=\rho(t, m)$, for $t>0$, we obtain

$$
\begin{aligned}
\frac{t}{\rho} & =\sum_{j \leqslant m} \frac{1}{j+\rho}+\sum_{j \leqslant m}\left(\frac{1}{a_{j}+\rho}-\frac{1}{j+\rho}\right) \\
& =\int_{1}^{m} \frac{d u}{u+\rho}+\mathrm{O}(1)=\log \frac{m+\rho}{1+\rho}+\mathrm{O}(1) .
\end{aligned}
$$

By virtue of

$$
m^{1-\varepsilon} \geqslant t \geqslant \frac{m \rho}{m+\rho},
$$

we have $\rho \ll m^{1-\varepsilon}$. Now (15) reduces to

$$
\frac{t}{\rho}=\log \frac{m}{1+\rho}+\mathrm{O}(1) .
$$

If $\rho \ll 1$, then $t / \rho=\log m+\mathrm{O}(1)$. The last equality is equivalent to (14).

In the remaining case $1 \ll \rho \ll m^{1-\varepsilon}$, where $m \geqslant m(\varepsilon)$ is sufficiently large, by (16),

$$
\log m+\mathrm{O}(1) \geqslant \frac{t}{\rho} \geqslant \varepsilon \log m+\mathrm{O}(1)
$$

Hence $\rho=B t / \log m$ with some $B=B(t, m)$, where $0<c(\varepsilon) \leqslant B \leqslant C(\varepsilon)$ for all $m \geqslant 3$ and some constants $c(\varepsilon)$ and $C(\varepsilon)$ depending on $\varepsilon$. Since

$$
\begin{aligned}
\log (1+\rho) & =\log \left(1+\frac{t}{\log m}\right)+\log \frac{\log m+B t}{\log m+t} \\
& =\log \left(1+\frac{t}{\log m}\right)+\mathrm{O}(1)
\end{aligned}
$$


from (16) we obtain the desired formula (14).

The lemma is proved.

We set $b_{j}:=a_{j}^{-1}=\mathrm{e}^{1 / j}-1$ and examine an analytic function $\varphi(z)$ which, for $|z|<a_{1}$ or $\Re z>0$, is defined by

$$
\varphi(z):=\sum_{j \leqslant m} \log \left(1+b_{j} z\right)=\log F(z, m) .
$$

Denote

$$
s_{r}=\left.\frac{\mathrm{d}^{r} \varphi\left(\rho \mathrm{e}^{w}\right)}{\mathrm{d} w^{r}}\right|_{w=0} .
$$

Lemma 2. In the above notation, if $1 \leqslant k \leqslant m^{1-\varepsilon}$, then

$$
\varphi(\rho)=k+\mathrm{O}(\rho)=k\left(1+\mathrm{O}\left(L^{-1}\right)\right) .
$$

If $|z-1| \leqslant(1+2 \rho) / 4 \rho$, then

$$
f(z):=\varphi(\rho z)-\varphi(\rho)=k(z-1)+\mathrm{O}\left(k L^{-1}|z-1|^{2}\right) .
$$

Moreover, $s_{1}=k$ and

$$
s_{r}=k\left(1+\mathrm{O}\left(L^{-1}\right)\right), \quad r \geqslant 2 .
$$

Proof. We will use the estimates $0<b_{j} \leqslant \min \{2, \mathrm{e} / j\}$ and $0<b_{j}-1 / j \leqslant 2 j^{-2}$ for $j \geqslant 1$. It suffices to take sufficiently large $m$.

In the proof of Lemma 1 , we observed that $\rho \leqslant C_{1}(\varepsilon) m^{1-\varepsilon}$. If $\rho \geqslant(2 \mathrm{e})^{-1}$, then

$$
\begin{aligned}
\varphi(\rho) & =\left(\sum_{2 \mathrm{e} \rho<j \leqslant m}+\sum_{j \leqslant 2 \mathrm{e} \rho}\right) \log \left(1+b_{j} \rho\right) \\
& =\rho \sum_{2 \mathrm{e} \rho<j \leqslant m} b_{j}+\mathrm{O}\left(\rho^{2} \sum_{2 \mathrm{e} \rho<j \leqslant m} \frac{1}{j^{2}}\right)+\mathrm{O}\left(\sum_{j \leqslant 2 \mathrm{e} \rho} \log (3 \mathrm{e} \rho / j)\right) \\
& =\rho \log \frac{m}{\rho+1}+\mathrm{O}(\rho) \\
& =k+\mathrm{O}(\rho)=k\left(1+\mathrm{O}\left(L^{-1}\right)\right) .
\end{aligned}
$$

In the last step we applied formula (14) and in the step before that we applied formula (16). The derived expression for $\varphi(\rho)$ also holds in the easier case $\rho<(2 \mathrm{e})^{-1}$. We omit the details.

To prove formula (18), we observe that

$$
\frac{b_{j} \rho|z-1|}{1+b_{j} \rho} \leqslant \frac{2 \rho}{1+2 \rho} \cdot \frac{1+2 \rho}{4 \rho}=\frac{1}{2}
$$

in the given region, therefore the function

$$
f(z)=\sum_{j \leqslant m} \log \left(1+\frac{b_{j} \rho(z-1)}{1+b_{j} \rho}\right)
$$


is analytic in it. Expanding the logarithm, we obtain

$$
\begin{aligned}
f(z) & =(z-1) \sum_{j \leqslant m} \frac{b_{j} \rho}{1+b_{j} \rho}+\mathrm{O}\left(\rho^{2}|z-1|^{2} \sum_{j \leqslant m} \frac{1}{j^{2}+\rho^{2}}\right) \\
& =k(z-1)+\mathrm{O}\left(\frac{\rho^{2}|z-1|^{2}}{\rho+1}\right) \\
& =k(z-1)+\mathrm{O}\left(k L^{-1}|z-1|^{2}\right) .
\end{aligned}
$$

To derive relations (19), it suffices to apply (18) and Cauchy's formula on the circumference $|w|=c$, where $c>0$ is chosen so that $\left|e^{w}-1\right| \leqslant c \mathrm{e}^{c} \leqslant 1 / 2 \leqslant(1+2 \rho) /(4 \rho)$.

The lemma is proved.

Remark. The argument mentioned in the last step actually yields the Taylor expansion of $f\left(\mathrm{e}^{w}\right)$ in the region $|w| \leqslant c$.

Lemma 3. There exists an absolute positive constant $c_{1}$ such that

$$
\left|F\left(\rho \mathrm{e}^{i \tau}, m\right)\right| \ll \exp \left\{-c_{1} k \tau^{2}\right\} F(\rho, m)
$$

uniformly in $1 \leqslant k \leqslant m^{1-\varepsilon}$ and $|\tau| \leqslant \pi$.

Proof. We apply the identity

$$
\frac{\left|1+x \mathrm{e}^{i \tau}\right|^{2}}{(1+x)^{2}}=1-\frac{2 x(1-\cos \tau)}{(1+x)^{2}}
$$

with $x=b_{j} \rho \leqslant \mathrm{e} \rho / j \leqslant 1 / 4$ for $j>4 \mathrm{e} \rho$ and obtain

$$
\begin{aligned}
\frac{\left|F\left(\rho \mathrm{e}^{i \tau}, m\right)\right|^{2}}{F(\rho, m)^{2}} & \leqslant \prod_{4 \mathrm{e} \rho \leqslant j \leqslant m}\left(1-\frac{2 b_{j} \rho(1-\cos \tau)}{\left(1+b_{j} \rho\right)^{2}}\right) \\
& \leqslant \exp \left\{\sum_{4 \mathrm{e} \rho<j \leqslant m} \log \left(1-\frac{2 b_{j} \rho(1-\cos \tau)}{\left(1+b_{j} \rho\right)^{2}}\right)\right\} \\
& \left.\leqslant \exp \left\{-\sum_{4 \mathrm{e} \rho<j \leqslant m} \frac{b_{j} \rho(1-\cos \tau)}{\left(1+b_{j} \rho\right)^{2}}\right)\right\} \\
& \leqslant \exp \left\{-\frac{4}{5}(1-\cos \tau) \sum_{4 \mathrm{e} \rho<j \leqslant m} \frac{b_{j} \rho}{1+b_{j} \rho}\right\} .
\end{aligned}
$$

Now, to involve $k$, using the definition of $\rho$ we complete the sum in the exponent by the quantity

$$
\sum_{j \leqslant 4 \mathrm{e} \rho} \frac{b_{j} \rho}{1+b_{j} \rho}=\mathrm{O}(\rho)=\mathrm{O}\left(\frac{k}{L}\right) .
$$

By virtue of the inequality $1-\cos \tau \geqslant 2 \tau^{2} / \pi^{2}$, we now obtain

$$
\frac{\left|F\left(\rho \mathrm{e}^{i \tau}, m\right)\right|^{2}}{F(\rho, m)^{2}} \leqslant \exp \left\{-\frac{8 \tau^{2}}{5 \pi^{2}} k\left(1+\mathrm{O}\left(\frac{1}{L}\right)\right)\right\}
$$


for $1 \leqslant k \leqslant m^{1-\varepsilon}$. This implies the desired estimate if $m \geqslant m(\varepsilon)$ is sufficiently large. For $3 \leqslant m \leqslant m(\varepsilon)$, the claim of Lemma 3 is trivial.

The lemma is proved.

Proof of Proposition 2. For $k=0$, its claim is evident therefore we assume that $k \geqslant 1$. Firstly, we separate a special case. If $\rho \leqslant 1 / 6$, then $|z|=1$ implies $|z-1| \leqslant 2 \leqslant(2 \rho+1) / 4 \rho$. Thus estimate (18) is at our disposal. Moreover, as we have observed in the proof of Lemma $1, k / \rho=\log m+\mathrm{O}(1)$ and $L \asymp \log m$. So, using Lemma 2, we obtain

$$
\begin{aligned}
q_{k}(m) & =\frac{F(\rho, m)}{\rho^{k} 2 \pi i} \int_{|w|=1} \frac{\exp \{f(w)\}}{w^{k+1}} \mathrm{~d} w \\
& =\frac{F(\rho, m)}{(\mathrm{e} \rho)^{k} 2 \pi i} \int_{|w|=1} \frac{\mathrm{e}^{k w}}{w^{k+1}}\left(1+\mathrm{O}\left(k L^{-1}|w-1|^{2}\right)\right) \mathrm{d} w \\
& =\frac{F(\rho, m)}{(\mathrm{e} \rho)^{k}}\left(\frac{k^{k}}{k !}+\mathrm{O}\left(\frac{k \mathrm{e}^{k}}{L} \int_{-\pi}^{\pi} \mathrm{e}^{-k(1-\cos \tau)}(1-\cos \tau) \mathrm{d} \tau\right)\right) .
\end{aligned}
$$

By virtue of $1-\cos \tau \asymp \tau^{2}$ for $|\tau| \leqslant \pi$, the last integral is of order $k^{-3 / 2}$. This and Stirling's formula yield the desired asymptotic formula in the selected case.

If $1 / 6 \leqslant \rho \ll m^{1-\varepsilon}$, then we can start with

$$
q_{k}(m)=\frac{F(\rho, m)}{\rho^{k} 2 \pi} \int_{-\pi}^{\pi} \exp \left\{f\left(\mathrm{e}^{i \tau}\right)-i k \tau\right\} \mathrm{d} \tau .
$$

Using the expansion of $f\left(\mathrm{e}^{i \tau}\right)$ mentioned in Remark after Lemma 2 , for $|\tau| \leqslant c$, we have

$$
f\left(\mathrm{e}^{i \tau}\right)=i k \tau-\frac{1}{2} s_{2} \tau^{2}-\frac{i}{6} s_{3} \tau^{3}+\mathrm{O}\left(k \tau^{4}\right) .
$$

Hence

$$
\exp \left\{f\left(\mathrm{e}^{i \tau}\right)-i k \tau\right\}=\mathrm{e}^{-s_{2} \tau^{2} / 2}\left(1-\frac{i s_{3} \tau^{3}}{6}+\mathrm{O}\left(k \tau^{4}+k^{2} \tau^{6}\right)\right)
$$

for $|\tau| \leqslant c k^{-1 / 3}$. Exploiting the symmetry of the term with $\tau^{3}$ we have

$$
\begin{aligned}
I_{1} & :=\frac{1}{2 \pi} \int_{|\tau| \leqslant c k^{-1 / 3}} \exp \left\{f\left(\mathrm{e}^{i \tau}\right)-i k \tau\right\} \mathrm{d} \tau \\
& =\frac{1}{2 \pi}\left(\int_{\mathbf{R}}-\int_{|\tau|>c k^{-1 / 3}}\right) \mathrm{e}^{-s_{2} \tau^{2} / 2} \mathrm{~d} \tau+\mathrm{O}\left(k^{-3 / 2}\right) \\
& =\frac{1}{\sqrt{2 \pi s_{2}}}+\mathrm{O}\left(k^{-3 / 2}\right)=\frac{1}{\sqrt{2 \pi k}}\left(1+\mathrm{O}\left(L^{-1}\right)\right) .
\end{aligned}
$$

In the last step we used (19) and the inequality $k \gg L$ following from $\rho \geqslant 1 / 6$.

Applying Lemma 3 we obtain

$$
\begin{aligned}
I_{2} & :=\int_{c k^{-1 / 3} \leqslant|\tau| \leqslant \pi} \frac{\left|F\left(\rho \mathrm{e}^{i \tau}, m\right)\right|}{F(\rho, m)} \mathrm{d} \tau \\
& \ll \int_{|\tau| \geqslant c k^{-1 / 3}} \mathrm{e}^{-c_{1} k \tau^{2}} \mathrm{~d} \tau \ll k^{-3 / 2} .
\end{aligned}
$$


Collecting the estimates of $I_{1}$ and $I_{2}$, we see that

$$
q_{k}(m)=\frac{F(\rho, m)}{\rho^{k}}\left(I_{1}+\mathrm{O}\left(I_{2}\right)\right)=\frac{F(\rho, m)}{\rho^{k} \sqrt{2 \pi k}}\left(1+\mathrm{O}\left(L^{-1}\right)\right) .
$$

Again Stirling's formula yields the desired result.

The proposition is proved.

\section{Proofs of Theorems and Corollaries}

The claim of Theorem 1 follows from Proposition 2. We will discuss only the remaining statements.

Proof of Corollary. The case $k=0$ is trivial. If $k \geqslant 1$, formula (7) follows from Proposition 2, (14), and (17).

To prove $(8)$, let us set $\rho=\rho(k, m)$ and $L=L(k, m)$. Applying Proposition 2 we approximate $F(\rho, m)$ by $F(k / L, m)$. That is available because of the inequality $\mid k /(\rho L)-$ $1 \mid \leqslant 1 / 2$ following from (14) provided that $m$ is sufficiently large. By (18) and (14), we have

$$
\begin{aligned}
\varphi(\rho) & =\varphi\left(\rho \frac{k}{\rho L}\right)-k\left(\frac{k}{\rho L}-1\right)+\mathrm{O}\left(\frac{k}{L}\left|\frac{k}{\rho L}-1\right|^{2}\right) \\
& =\varphi\left(\frac{k}{L}\right)-k \log \left(\frac{k}{\rho L}\right)+\mathrm{O}\left(\frac{k}{L^{2}}\right) .
\end{aligned}
$$

Inserting this into the equality in Proposition 2 we complete the proof of (8).

Proof of Theorem 2. Observe that, for $1 \leqslant k-1 \leqslant t \leqslant k \leqslant m^{1-\varepsilon}$, we have $L(t, m-1)=$ $L(k, m-1)+\mathrm{O}(1 / \log m)$ and $L(k, m-1)=L(k, m)+\mathrm{O}(1 / m)$ therefore afterwards, for different arguments, we may use $L=L(k, m)$. Denote $r(t):=\rho(t, m-1), r_{0}=r(k-1)$, and $r_{1}=r(k)$. Then, by Proposition 2,

$$
\begin{aligned}
\frac{d_{k+1}(m)}{d_{k}(m)} & =\frac{q_{m-1}(k)}{q_{m-1}(k-1)} \\
& =\frac{F\left(r_{1}, m-1\right)}{F\left(r_{0}, m-1\right)} \frac{r_{0}^{k-1}}{r_{1}^{k}} \frac{1}{\mathrm{e}}\left(\frac{k}{k-1}\right)^{k-1}\left(1+\mathrm{O}\left(\frac{1}{L}\right)\right)
\end{aligned}
$$

for $k \geqslant 1$. Set

$$
K(t)=\log F(r(t), m-1)-t \log r(t)
$$

We have

$$
\frac{d_{k+1}(m)}{d_{k}(m)}=\exp \left\{\int_{k-1}^{k} K^{\prime}(t) d t-1+(k-1) \log \left(\frac{k}{k-1}\right)+\mathrm{O}\left(\frac{1}{L}\right)\right\} .
$$


By the definitions,

$$
\begin{aligned}
K^{\prime}(t) & =r^{\prime}(t)\left(\frac{F^{\prime}(r(t), m-1)}{F(r(t), m-1)}-\frac{t}{r(t)}\right)-\log r(t) \\
& =-\log r(t)=\log L-\log t+\mathrm{O}\left(L^{-1}\right) .
\end{aligned}
$$

Inserting this expression into the previous equality and integrating we obtain the desired result (10).

To prove (11), we first restrict the region to $1 \leqslant k \leqslant \sqrt{m}$ where all obtained remainder term estimates contain absolute constants. Hence, by virtue of (10), we can find absolute positive constants $C_{2}$ and $C_{3}$ such that $d_{k}(m)$ is increasing for $1 \leqslant k \leqslant \log m-C_{2}$ and decreasing for $\log m+C_{3} \leqslant k \leqslant \sqrt{m}$. If $k=\log m+\mathrm{O}(1)$, then

$$
L(k, m)=\log m+\mathrm{O}(1), \quad \rho=\rho(k, m)=1+\mathrm{O}\left(\log ^{-1} m\right),
$$

and, by (18) applied with $z=1 / \rho$,

$$
\varphi(\rho)-k \log \rho-\varphi(1) \ll 1 / \log m .
$$

This and Proposition 2 imply

$$
\begin{aligned}
P_{m}(k) & =\frac{\exp \{\varphi(\rho)-k \log \rho-\varphi(1)\}}{W(k)}\left(1+\mathrm{O}\left(\frac{1}{\log m}\right)\right) \\
& =\frac{1}{\sqrt{2 \pi \log m}}\left(1+\mathrm{O}\left(\frac{1}{\log m}\right)\right)
\end{aligned}
$$

for $k=\log m+\mathrm{O}(1)$. The same holds for $P_{m-1}(k-1)$. Recalling (9) we obtain

$$
\max _{1 \leqslant k \leqslant \sqrt{m}} d_{k}(m)=\frac{1}{m \sqrt{2 \pi \log m}}\left(1+\mathrm{O}\left(\frac{1}{\log m}\right)\right) .
$$

It remains to estimate $d_{k}(m)$ for $\sqrt{m} \leqslant k \leqslant m$. Differentiating the function $F(z, m)$ we have the estimate

$$
\begin{aligned}
q_{k}(m) & \leqslant \frac{1}{k !}\left(\sum_{j \leqslant m}\left(\mathrm{e}^{-1 / j}-1\right)\right)^{k} \\
& \leqslant \exp \left\{k \log \left(2 \log m+C_{4}\right)-k \log k+\mathrm{O}(k)\right\} \\
& \ll \exp \{-(1 / 3) \sqrt{m} \log m\}
\end{aligned}
$$

which shows that the maximum of $d_{k}(m)=q_{k-1}(m-1) / F(1, m-1)$ over $1 \leqslant k \leqslant m$ is given by (20).

The theorem is proved.

Proof of Theorem 3. Let

$$
g(z)=\sum_{j \geqslant 1}\left(\log \left(1+b_{j} z\right)-\frac{b_{j} z}{1+b_{j}}\right)+C z, \quad G(z)=\mathrm{e}^{g(z)} .
$$


The function $g(z)$ has an analytic continuation to the region $\mathbf{C} \backslash\left[-(\mathrm{e}-1)^{-1},-\infty\right)$, however, we prefer to use it as a function of real variable. Observe that

$$
G(u)=\mathrm{e}^{\gamma}\left(1+C(u-1)+\mathrm{O}\left((1-u)^{2}\right)\right) .
$$

for $u \in[0, T]$, where $T>0$ is an arbitrary fixed number and the constant in $\mathrm{O}(\cdot)$ depends on $T$. For such $u$, using elementary inequalities we can rewrite

$$
\begin{aligned}
F(u, y) & =\exp \left\{\sum_{j \leqslant y}\left(\log \left(1+b_{j} u\right)-\frac{b_{j} u}{1+b_{j}}\right)+u \sum_{j \leqslant y}\left(\frac{b_{j}}{1+b_{j}}-\frac{1}{j}\right)+u \sum_{j \leqslant y} \frac{1}{j}\right\} \\
& =G(u) y^{u}(1+\mathrm{O}(1 / y)),
\end{aligned}
$$

where the remainder depends on $T$ only.

Let $k \geqslant 1$ be arbitrary, $0 \leqslant l \leqslant k$, and let $y \geqslant 3$ be such that $k / 10 \leqslant \log y \leqslant 10 k$. Then $|L(l, y)-\log y| \leqslant \log 11$ and

$$
\frac{l}{L(l, y)}=\frac{l}{\log y}\left(1+\mathrm{O}\left(\frac{1}{\log y}\right)\right)
$$

where the constant in $\mathrm{O}(\cdot)$ is absolute. From formula (8) with $y$ instead of $m$ and (22) with $u=l / L(l, y)$, we obtain

$$
\begin{aligned}
q_{l}(y) & =F(1, y) P_{y}(l) \\
& =F\left(\frac{l}{L(l, y)}, y\right)\left(\frac{L(l, y)}{\mathrm{e}}\right)^{l} \frac{1}{l !}\left(1+\mathrm{O}\left(\frac{1}{k}\right)\right) \\
& =G\left(\frac{l}{L(l, y)}\right) \exp \left\{\frac{l \log y}{L(l, y)}+l \log L(l, y)-l\right\} \frac{1}{l !}\left(1+\mathrm{O}\left(\frac{1}{k}\right)\right) \\
& =G\left(\frac{l}{\log y}\right) \frac{(\log y)^{l}}{l !}\left(1+\mathrm{O}\left(\frac{1}{k}\right)\right)
\end{aligned}
$$

for $0 \leqslant l \leqslant k$ with an absolute constant in the remainder term. In the exponent, we have used the second order approximation of the logarithmic function.

If $k / 10 \leqslant \log y \leqslant 10 k$, then recalling (21) and (22), we arrive at

$$
\begin{aligned}
d\left(j_{k}(\sigma)>y\right) & =\frac{1}{F(1, y)} \sum_{0 \leqslant l<k} q_{l}(y) \\
& =\sum_{0 \leqslant l<k} \frac{(\log y)^{l}}{l ! y}\left\{1+C\left(\frac{l}{\log y}-1\right)+\mathrm{O}\left(\frac{1}{k}+\left(\frac{l}{\log y}-1\right)^{2}\right)\right\} \\
& =\sum_{0 \leqslant l<k} \frac{(\log y)^{l} \mathrm{e}^{-\log y}}{l !}-C \frac{(\log y)^{k-1}}{(k-1) ! y}+\mathrm{O}\left(\frac{1}{k}\right) .
\end{aligned}
$$

Consequently, if $|x| \leqslant k^{1 / 6}$ and $k \geqslant 3$, the last equality for $y=\mathrm{e}^{k+x \sqrt{k}}$ implies

$$
\begin{aligned}
d\left(\log j_{k}(\sigma)>k+x \sqrt{k}\right)= & S_{k}(\log y)-\frac{(k+x \sqrt{k})^{k} \mathrm{e}^{-k-x \sqrt{k}}}{k !} \\
& \quad-C \frac{(k+x \sqrt{k})^{k-1}}{(k-1) !} \mathrm{e}^{-k-x \sqrt{k}}+\mathrm{O}\left(\frac{1}{k}\right),
\end{aligned}
$$


where, by Lemma 2.1 from [5],

$$
S_{k}(\log y):=\sum_{0 \leqslant l \leqslant k} \frac{(k+x \sqrt{k})^{l} \mathrm{e}^{-k-x \sqrt{k}}}{l !}=1-\Phi(x)+\frac{\left(2+x^{2}\right) \mathrm{e}^{-x^{2} / 2}}{3 \sqrt{2 \pi k}}+\mathrm{O}\left(\frac{1}{k}\right) .
$$

For the other terms in (23), Stirling's formula yields

$$
\frac{(k+x \sqrt{k})^{k} \mathrm{e}^{-k-x \sqrt{k}}}{k !}=\frac{\mathrm{e}^{-x^{2} / 2}}{\sqrt{2 \pi k}}\left(1+\mathrm{O}\left(\frac{|x|^{3}}{\sqrt{k}}\right)\right)\left(1+\mathrm{O}\left(\frac{1}{k}\right)\right)=\frac{\mathrm{e}^{-x^{2} / 2}}{\sqrt{2 \pi k}}+\mathrm{O}\left(\frac{1}{k}\right)
$$

provided that $|x| \leqslant k^{1 / 6}$. The last quantity on the right-hand side is also equal to the factor of $-C$ in (23). Inserting these estimates into (23) we obtain the desired result (12) for $|x| \leqslant k^{1 / 6}$ and $k \geqslant 3$.

If $x \geqslant k^{1 / 6}$, by monotonicity of $x \mapsto d\left(\log j_{k}(\sigma)>k+x \sqrt{k}\right)$ and the just proved relation,

$$
d\left(\log j_{k}(\sigma)>k+x \sqrt{k}\right) \leqslant d\left(\log j_{k}(\sigma)>k+k^{2 / 3}\right) \ll k^{-1} .
$$

Consequently, formula (12) trivially holds in this region. Similarly, if $x \leqslant-k^{1 / 6}$, to verify equality (12), we can use the estimate

$$
d\left(\log j_{k}(\sigma) \geqslant k+x \sqrt{k}\right) \leqslant d\left(\log j_{k}(\sigma) \geqslant k-k^{2 / 3}\right) \ll k^{-1} .
$$

The theorem is proved.

Acknowledgement. The author thanks an anonymous referee for his helpful advises how to improve the presentation of the paper.

\section{References}

[1] R. Arratia and S. Tavaré, The cycle structure of random permutations, Ann. Probab., 1992, 20, 3, 1567-1591.

[2] R. Arratia, A.D. Barbour and S. Tavaré, Logarithmic Combinatorial Structures: a Probabilistic Approach, EMS Monographs in Mathematics, EMS Publishing House, Zürich, 2003.

[3] G.J. Babu and E. Manstavičius, Brownian motion and random permutations, Sankhyā, A, 1999, 61, 3, 312-327.

[4] K. Borovkov and D. Pfeifer, On improvements of the order of approximation in the Poisson limit theorem, J. Appl. Probab., 1996, 33, 146-155.

[5] J.-M. De Koninck and G. Tenenbaum, Sur la loi de répartition du $k$-ième facteur premier d'un entier, Math. Proc. Cambridge Phil. Soc., 2002, 113, 133-191.

[6] P. Erdős and G. Tenenbaum, Sur les densités de certaines suites d'entiers, Proc. London Math. Soc., 1989, 59, 3, 417-438.

[7] H.-K. Hwang, Large deviations of combinatorial distributions. II. Local limit theorems, Ann. Appl. Probab., 1998, 8, 163-181. 
[8] H.-K. Hwang, Asymptotics of Poisson approximation to random discrete distributions: an analytic approach, Advances in Appl. Probab., 1999, 31, 448-491.

[9] V.F. Kolchin, A problem of the allocation of particles in cells and cycles of random permutations, Teor. Veroyatnost. i Primenen., 1971, 16, 1, 67-82 (Russian).

[10] E. Manstavičius, Semigroup elements free of large prime factors. In: New Trends in Probability and Statistics, vol 2. Analytic and Probabilistic Methods in Number Theory, (F.Schweiger, E.Manstavičius, Eds), TEV/Vilnius, VSP/Utrecht, 1992, 135153.

[11] E. Manstavičius, Remarks on the semigroup elements free of large prime factors, Lith. Math. J., 1992, 32, 4, 400-410.

[12] E. Manstavičius, Additive and multiplicative functions on random permutations, Lith. Math. J., 1996, 36, 4, 400-408.

[13] E. Manstavičius, The law of iterated logarithm for random permutations, Lith. Math. J., 1998, 38, 160-171.

[14] E. Manstavičius, Iterated logarithm laws and the cycle lengths of a random permutation, Trends Math., Mathematics and Computer Science III, Algorithms, Trees, Combinatorics and Probabilities, M.Drmota et al (Eds), Birkhauser Verlag, Basel, 2004, 39-47.

[15] E. Manstavičius, Asymptotic value distribution of additive function defined on the symmetric group, The Ramanujan J., 2008, 17, 259-280.

[16] D. Panario and B. Richmond, Smallest components in decomposable structures: ExpLog class, Algorithmica, 2001, 29, 205-226.

[17] V.V. Petrov, Sums of Independent Random Variables, Springer Verlag, Berlin, 1975. 